\title{
Durable Response with Single-Agent Pembrolizumab in a Patient with Metastatic Melanoma
}

\author{
Sanju Cyriac ${ }^{1}$ Ajith Toms ${ }^{2}$ Sunitha Thomas ${ }^{3}$ \\ ${ }^{1}$ Department of Medical Oncology, Rajagiri Hospital, Kochi, Kerala, \\ India \\ 2 Department of Radiology, Rajagiri Hospital, Kochi, Kerala, India \\ ${ }^{3}$ Department of Pathology, Rajagiri Hospital, Kochi, Kerala, India
}

South Asian J Cancer 2022;11(1):82-83.

Advanced melanoma, with a poor prognosis and high propensity to metastasize, is the most belligerent cutaneous malignancy. ${ }^{1}$ Mortality due to the disease is increasing in spite of early detection and appropriate management. Melanoma contributes to roughly $80 \%$ of all skin cancer-related deaths as per WHO report. ${ }^{2}$ Advanced melanoma has been transformed into a new oncological model for treating solid cancers through increased biological understanding and access to innovative therapeutic modalities. In particular, immune checkpoint blockers have shown a major success in treating advanced melanoma. ${ }^{3}$

Here, we are presenting the case of a patient who was diagnosed with malignant melanoma as well as discussing the importance of pembrolizumab.

\section{Case Report}

The patient was a 64-year-old housewife who was diagnosed with malignant melanoma of her right sole in 2012. She underwent wide local excision and split skin graft for the same. Postoperatively, Histopathology report was suggested, and she was under regular follow-up.

In 2016, she came back with a bleeding lesion at the site of tumor ( - Fig. 1). Clinically, it was a $1 \mathrm{~cm}$ lesion. There were no satellite lesions, in-transit nodules, or inguinal lymph nodes.

Staging CT chest revealed multiple bilateral lung metastases (-Fig. 2). Biopsy from the lesion suggested melanoma. BRAF mutation was negative, and immunohistochemistry revealed PDL1 of $15 \%$.

The patient was offered immunotherapy, and single-agent pembrolizumab $100 \mathrm{mg}$ was given. She was noted to have

DOI https://doi.org/10.1055/s-0041-1731911 ISSN 2278-330X

How to cite this article: Cyriac $S$, Toms A, Thomas $S$ Durable Response with Single-Agent Pembrolizumab in a Patient with Metastatic Melanoma South Asian J Cancer 2022;11(1):82-83.
Address for correspondence Sanju Cyriac, MD, DM, DNB, Department of Medical Oncology, Rajagiri Hospital, Aluva, Kochi 683112, Kerala, India (e-mail: drsanpan80@yahoo.com).

clinical response with every cycle. At the end of eight cycles, she showed complete response (CR) of the primary lesion (-Fig. 3). The metastatic sites showed partial response. Unfortunately, she had vitiligo as immune-mediated adverse event and hence we were forced to stop the treatment. However, she never had a progression of the local site till date and is still coming for follow-up.
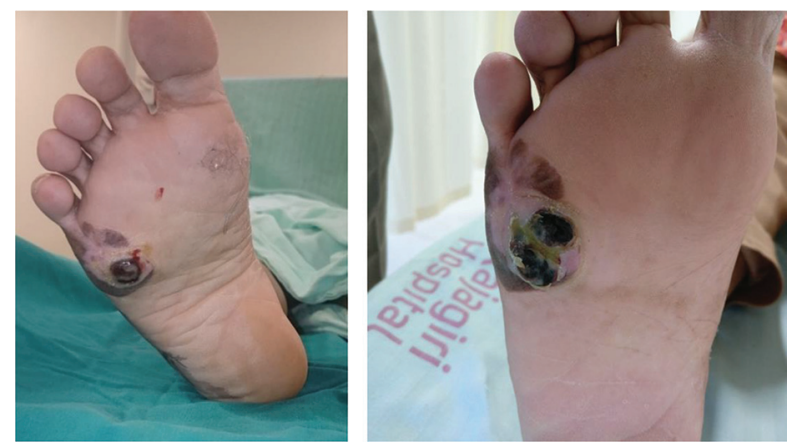

Fig. 1 Bleeding lesion at the site of tumor.
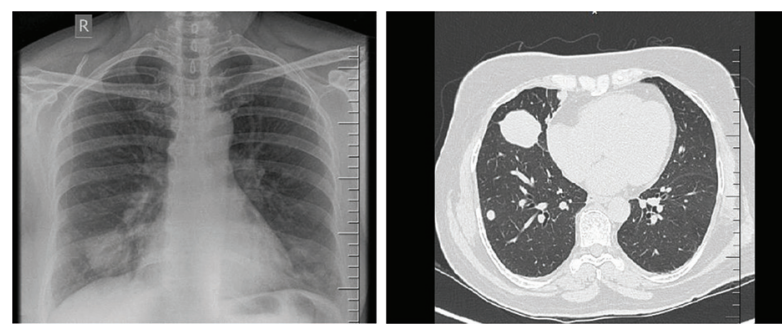

Fig. 2 CT chest.

(c) 2021. MedIntel Services Pvt Ltd. All rights reserved.

This is an open access article published by Thieme under the terms of the Creative Commons Attribution-NonDerivative-NonCommercial-License, permitting copying and reproduction so long as the original work is given appropriate credit. Contents may not be used for commercial purposes, or adapted, remixed, transformed or built upon. (https://creativecommons.org/licenses/by-nc-nd/ 4.0/)

Thieme Medical and Scientific Publishers Pvt. Ltd., A-12, 2nd Floor, Sector 2, Noida-201301 UP, India 


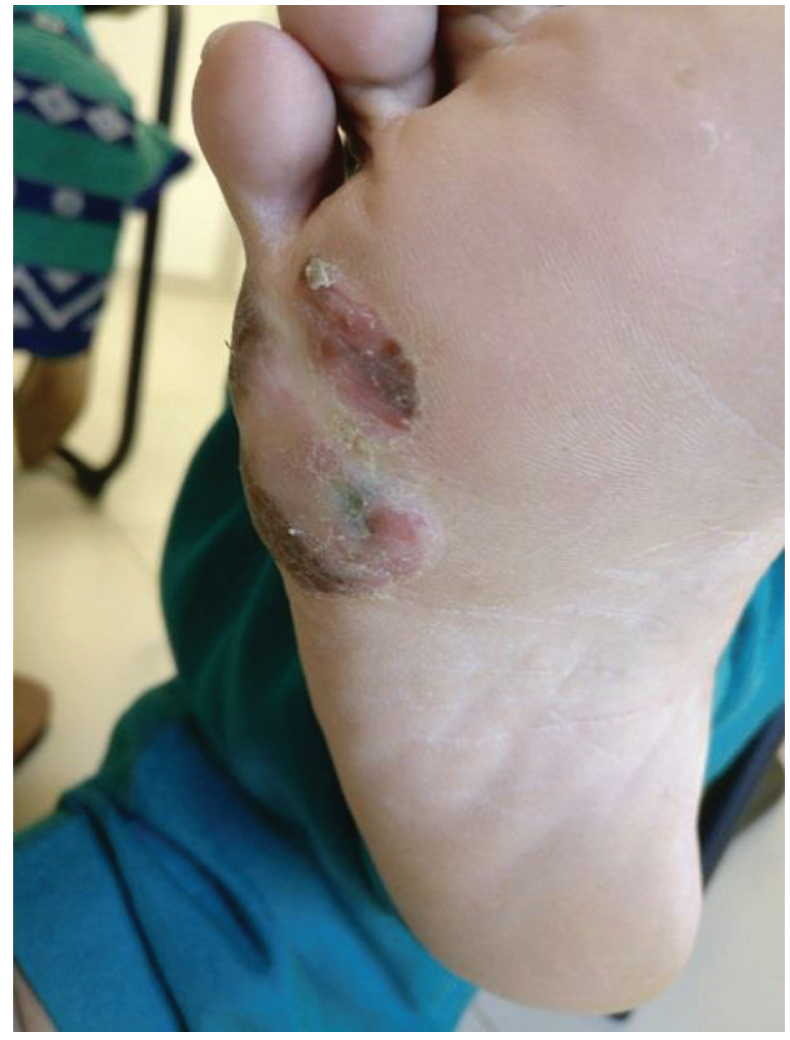

Fig. 3 Lesion after eight treatment cycles.

\section{Discussion}

Immunotherapy has changed the way melanoma is treated. In particular, checkpoint inhibitors increase the survival rate for patients with metastatic melanoma. ${ }^{4}$ The anti-programmed death 1 (PD-1) monoclonal antibody pembrolizumab, which blocks binding of PD-1 to its ligands PD-L1 and PD-L2, showed response rates of 30 to $40 \%$ and median overall survival of $>2$ years in patients with metastatic melanoma. ${ }^{5}$ The 24-month disease-free survival (DFS) was estimated to be $90.9 \%$ after a median follow-up of 43 months and a median follow-up of 30 months from the time of first declaration of CR. The risk for disease progression or death after CR and discontinuation of pembrolizumab was similarly low (24-month DFS was estimated to be $85.8 \%$ for all patients who stopped treatment of any reason other than progressive disease and was $89.9 \%$ for those who discontinued treatment after CR for observation). ${ }^{5}$

In this article, we had presented a case of malignant melanoma who responded to single-agent pembrolizumab and is still keeping the response in accordance with the concept of immune memory. The T cells, activated with PD-1 inhibition, can continue the fight against cancer cells in spite of stopping the drug.

\section{Conclusion}

Single-agent pembrolizumab is an effective treatment option in patients with metastatic melanoma, which showed durable complete remission even after discontinuation of treatment.

How to activate the immune memory and how can we recognize whether immune memory is activated are the two crucial questions to be answered in immunotherapy treatment era. If understood well, we can give finite cycles for our patients, thereby saving lots of money.

Conflict of Interest

None declared.

\section{References}

1 Tsur N, Kogan Y, Avizov-Khodak E, et al. Predicting response to pembrolizumab in metastatic melanoma by a new personalization algorithm. J Transl Med 2019;17(01):338

2 Burns MC, O'Donnell A, Puzanov I. Pembrolizumab for the treatment of advanced melanoma. Expert Opin Orphan Drugs 2016;4 (08):867-873

3 Diamantopoulos P, Gogas H. Melanoma immunotherapy dominates the field. Ann Transl Med 2016;4(14):269

4 Robert C, Ribas A, Hamid O, et al. Durable complete response after discontinuation of pembrolizumab in patients with metastatic melanoma. J Clin Oncol 2018;36(17):1668-1674

5 Cancer Research Institute. Immunotherapy for Melanoma. Accessed August 20, 2020 at: https://www.cancerresearch.org/immunotherapy/cancer-types/melanoma 